\title{
Special issue on microscopic image processing
}

\author{
Kivanc Kose · Rengul Cetin-Atalay · A. Enis Cetin
}

Received: 20 October 2014 / Accepted: 23 October 2014 / Published online: 4 November 2014

(C) Springer-Verlag London 2014

Microscopic images play an essential role in detection and diagnosis of numerous diseases. For example, analysis of histology images of the human tissue biopsies remains the most reliable way of diagnosis and grading cancer. On the other hand, there is significant inter- and intra-rater variability in the grading and diagnosis of cancer from histology slides by clinicians. Computational histopathology can assist the pathologists in making the grading and diagnosis reproducible, by providing useful quantitative measures from histology images of a patient suspected or diagnosed of having cancer [1]. Similarly, in cellular biology, live cell fluorescence imaging made possible by the rapid advances of fluorescent microscopy opens new avenues for microscopic image processing. It is possible to give many similar examples.

In this special issue, we bring together six recent research articles on microscopic image processing and analysis. The subject topics of papers cover a diverse set of topics related to microscopic images processing such as creating efficient representations, segmentation of diagnostically significant structures, and automated classification. Even if the targeted applications are diverse, the main goal of all the algorithms was similar; they describe automated analysis methods for

K. Kose $(\bowtie)$

Dermatology Service, Memorial Sloan Kettering Cancer Center,

New York, NY, USA

e-mail: kkoseug@gmail.com

R. Cetin-Atalay

Cancer Systems Biology Laboratory, Graduate School of Informatics, Middle East Technical University, Ankara, Turkey

\section{A. E. Cetin}

Electrical and Electronics Engineering Department, Bilkent University, Ankara, Turkey microscopic images in a rapid and quantitative manner to help clinicians for more reliable diagnosis.

In "Automatic Panorama with Auto-focusing based on Image Fusion for Microscopic Imaging System" [2], Dogan et al. discuss the problem of imaging large tissue slides in an automated fashion. Field-of-view extension through mosaicing is an important technique in microscopic image processing, because most of the time, the clinicians do not rely their diagnosis on limited small field-of-view images generated by the microscopes. Typically, they either sift through the tissue sample by manually sliding it under the microscope objective or use expensive slide scanners to obtain large field-of-view digital images. In their study, Dogan et al. developed a color image fusion-based autofocus system followed by an automated microscopic image mosaicing method that can create high-resolution, large field-of-view images of the sample tissue using a low-cost system. The system enables quantitative imaging by minimizing the user input for focus purposes as well as it speeds up the imaging process. They showed the feasibility of their system on bright-field microscopy images of Mycobacterium tuberculosis in specimen of ZiehlNeelsen-stained sputum smears.

Segmentation of microscopic images is another area that computational methods are making an impact on. In many cases, the clinical diagnosis in histology relies on making quantifiable measurements from the microscopic images, such as counting number of a specific cell type or measuring their shape and sizes. Considering that a single microscopic image contains several of these structures, making such measurements manually, in a quantitative manner, is laborintensive if not infeasible. However, computer-aided segmentation algorithms are ideally suited for such operations as they rely on objective computational measurements. For example, in "A fluid dynamics based deformable model for segmentation of cervical cell images" [3], Cengizler et al. present 
a novel deformable model for unsupervised segmentation of cervical cells within Pap-smear images. They first segment the background and the cell nuclei. The extracted nuclei are then used as the initial seeds for their fluid mechanics-based model, which can be solved using Navier-Stokes propagation equations to delineate the borders of cervical cells.

In "Using Adaptive Neuro-Fuzzy Inference Systems for The Detection of Centroblasts In Microscopic Images of Follicular Lymphoma" [4], Dimitropoulos et al. present a framework for automatic detection of centroblasts (CB) in thin slices of follicular lymphomas (FL). This is an important problem in grading of FL as the clinicians determine the aggressiveness of the FL tumor by quantifying the number of the centroblasts with the biopsized tissue. Authors first segment the FL images into 3 basic cytological components: blood cells, nuclei, and extracellular material. After eliminating the blood cells and the extracellular material, the touching nuclei in the remaining image are split using a Gaussian mixture-based model. The resulting potential nuclei areas are then fed into an adaptive neuro-fuzzy inference system to be classified as cells. The framework yielded promising results with an average detection rate of $90.35 \%$.

With the help of new imaging technologies nowadays, the clinicians can go further beyond 2D imaging of thin tissue slices and perform depth imaging through sectioning. In this way, they can now be able to examine cellular structures in 3D. On the other hand, the amount of the generated data and the necessary labor force to process this data manually has increased substantially. In "GRED: Graph-Regularized 3D Shape Reconstruction from Highly Anisotropic and Noisy Images" [5], Widmer et al. look into the problem of automatically segmenting the cellular nuclear structures in highly anisotropic and noisy 3D fluorescent microscopic data. They aim to enable biologists to perform high-throughput quantitative experiments in fluorescence microscopy. Widmer et al. adapt graph-regularized transfer learning to the problem of parameter fitting in several layers and combine it with a robust loss function in order to minimize the need for manual post-processing. They experimentally validate the success of their results against commercially available software as well as the ground truth segmentation by the expert clinicians.

Such segmentation algorithms can be used as a preprocessing stage in many diagnostic classification frameworks. For example, in "Random Forest Based Tuberculosis Bacteria Classification in Images of ZN-stained Sputum Smear Samples" [6] Ayas et al. first apply an random forest (RF)-based segmentation on microscopic images of ZiehlNeelsen-stained sputum smears. They extract local color distribution around each pixel and use them as feature representations. A RF-based segmentation model together with these color features is then used for classifying each pixel as background or potential tuberculosis (TB). Then, potential TB pixels are grouped together using connected com- ponent analysis. At the final stage, the authors extract novel appearance-based features from each TB region and feed them into a RF-based learning model, which classifies them into TB or non-TB classes. The sensitivity and specificity of the segmentation model are 75.77 and $96.97 \%$, respectively. The classification model achieved sensitivity and specificity figures of 89.34 and $62.89 \%$, respectively, for the identification of bacilli objects.

In "Multi-scale Directional Filtering Based Method for Follicular Lymphoma (FL) Grading" [7], Bozkurt et al. look into the problem of automatically grading FL. Traditionally, FL grading is conducted by a clinician by counting the number of the centroblasts in a slide and calculating the centroblast density in a tissue slide. But obviously, performing such a task manually is both labor-intensive and prone to errors. Alican et al. observed that centroblast density in a tissue slide can effectively be described using textural patterns. Therefore, instead of counting the centroblasts individually, which is in itself another segmentation problem, they analyze the texture formed by centroblasts and conduct grading through texture classification. In this study, they developed a novel multiscale direction filtering-based scheme for extracting textural features, which are eventually be fed into a support vector machine (SVM)-based learning model for classification. They also compare their results against other well-known textural representation such as textons, complex wavelets, contourlets, and curvelets. Authors show that this novel texture representation model results in the best accuracy in classification among all the other textural representations. Moreover, the authors also present a runtime comparison between all the feature extraction methods used in the study and showed that their multiscale direction filteringbased scheme runs faster than all the other methods.

As editors, we believe that such special issues are very important in bridging the gap between the clinicians and engineers and computer scientists. Clinicians are able to see that their labor-intensive and qualitative procedures are automated through quantitative image analysis tools. Computer scientists and engineers can get feedback from clinicians, improve their algorithms, and discover new research opportunities. In this sense, we hope that the research efforts presented in this special issue will inspire similar research and eventually will help to pave the road for much faster, reliable, and also robust diagnosis and prognosis. This, in turn, will lead to a better patient care.

\section{References}

1. Gurcan, M.N., Boucheron, L.E., Can, A., Madabhushi, A., Rajpoot, N.M., Yener, B.: Histopathological image analysis: a review. IEEE Rev. Biomed. Eng. 2, 147-171 (2009)

2. Dogan, H., Ekinci, M.: Automatic panorama with auto-focusing based on image fusion for microscopic imaging system. SIViP, Spe- 
cial Issue on Microscopic Image Processing. 8(S1), (2014). doi:10. 1007/s11760-014-0717-5

3. Cengizler, C., Guven, M., Avci, M.: A fluid dynamics based deformable model for segmentation of cervical cell images. SIViP. 8(S1), (2014). doi:10.1007/s11760-014-0719-3

4. Dimitropoulos, K., Michail, E., Koletsa, T., et al.: Using adaptive neuro-fuzzy inference systems for the detection of centroblasts in microscopic images of follicular lymphoma. SIViP. 8(S1), (2014). doi:10.1007/s11760-014-0688-6
5. Widmer, C., Heinrich, S., Drewe, P., et al.: GRED: graph-regularized 3D shape reconstruction from highly anisotropic and noisy images. SIViP. 8(S1), (2014). doi:10.1007/s11760-014-0694-8

6. Ayas, S., Ekinci, M.: Random forest based tuberculosis bacteria classification in images of ZN-stained sputum smear samples. SIViP. 8(S1), (2014). doi:10.1007/s11760-014-0708-6

7. Bozkurt, A., Suhre, A., Cetin, A.E.: Multi-scale directional filtering based method for follicular lymphoma grading. SIViP. 8(S1), (2014). doi:10.1007/s11760-014-0681-0 